Мрочек А.Г., Вайханская Т.Г., Фролов А.В., Воробьев А.П., Мельникова О.П., Гуль Л.М., Сидоренко И.В., Боровкова Л.В.

ИДЕНТИФИКАЦИЯ

ЭЛЕКТРОКАРДИОГРАФИЧЕСКИХ

ПРЕДИКТОРОВ ЭЛЕКТРИЧЕСКОЙ

НЕСТАБИЛЬНОСТИ МИОКАРДА

Республиканский научно-практический чентр

«Кардиология»

2. Минск, Беларусь

Mrochek A.G., Vaikhanskaya T.G., Frolov A.V., Vorobiev A.P., Melnikova, O.P., Guel L.M., Sidorenko I.V., Borovkova L.V.

\title{
IDENTIFICATION OF THE ELECTROCARDIOGRAPHIC PREDICTORS OF THE MYOCARDIAL ELECTRICAL INSTABILITY
}

\section{PEЗЮME}

Целью работы является разработка и клиническая апробация компьютерной программы для идентификации комплекса прогностических маркеров внезапной сердечной смерти: альтернации Т-зубца, длительности и дисперсии интервала QT, турбулентности сердечного ритма (HRT). Материал и методы: 79 больных с кардиомиопатиями и жизнеопасными желудочковыми аритмиями и 30 пациентов без нарушений ритма. Использовали прецизионные цифровые методы обработки ЭКГ-сигнала. Результаты: получены достоверно высокие значения альтернации Т, дисперсии QT и турбулентности ритма у больных с желудочковыми аритмиями $(p<0,01)$. Заключение: выявление комплекса предикторов внезапной сердечной смерти необходимо для своевременной стратификации риска желудочковых тахиаритмий и выбора оптимальной лечебной тактики у пациентов с кардиомиопатией.

Ключевые слова: альтернация Т-зубца, дисперсия QT, программа, турбулентность сердечного ритма, электрокардиография.

\section{SUMMARY}

The aim is to develop and clinical test of the novel computer program for the identification of the prognostic markers for sudden cardiac death. Among them are T-wave alternans, duration and dispersion of the interval QT, heart rate turbulence. Material and methods: 79 pts with cardiomyopathy and life-threatening ventricular arrhythmias and 30 pts without arrhythmias. The methods of the precision digital processing of the ECG signal are used. Results: The significantly higher values of T-wave alternans, QT dispersion and heart rate turbulence in pts with ventricular arrhythmias were obtained $(p<0,01)$. Conclusion: The identification of the predictors of sudden cardiac death is necessary for timely risk stratification and choosing the treatment strategy in pts with cardiomyopathy.

Keywords: dispersion of the QT interval, electrocardiography, heart rate turbulence, T-wave alternans. 


\section{Контактная информация:}

\section{Кафедра госпитальной терапии №1 МгМСу, г Москва}

Мрочек Александр Геннадьевич - академик, директор РНПЦ“Кардиология”, тел.(017) 2087266

\section{Вайханская Татьяна Геннадьевна}

- К.М.Н., С.Н.с. РНПЦ “Кардиология”, тел.(017) 2861956

Фролов Александр Владимирович

Воробьев Анатолий Павлович

Мельникова Ольга Петровна

Гуль Людмила Михайловна

\section{Сидоренко Ирина Владимировна}

Боровкова Людмила Васильевна

\section{Республиканский научно- практический центр “Кардиология”}

Отв. за переписку: Фролов А.В.

- д.б.н., завлаб. РНПЦ “Кардиология”, тел.(017) 2861956

- с.н.с. РНПЦ “Кардиология”, тел.(017) 2560523

- с.н.с. РНПЦ “Кардиология”, тел.(017) 2560523

- н.с. РНПЦ “Кардиология”, тел.(017) 2560523

- врач РНПЦ “Кардиология”, тел.(017) 2073762

- зав.оперблоком РНПЦ“Кардиология”, тел.(017) 2085761

220036 г. Минск, ул. Р. Люксембург, 110

220036 г. Минск, ул.Р.Люксембург, 110, факс. (017) 2560523 E-mail:Frolov.Minsk@gmail.com
Проблема внезапной сердечной смерти (BCC) остается одной из актуальных в современной кардиологии и оценка риска ее развития - чрезвычайно сложная задача в медицине, интерес к которой со временем только возрастает. Несмотря на последние достижения в диагностике и лечении болезней сердца, доля ВСС по причине фатальных желудочковых аритмий остается высокой и достигает $11 \%$ в общей структуре смертности от сердечно-сосудистых заболеваний [1]. Поэтому активно продолжаются изучение и поиск новых прогностических факторов риска ВСС. Основными пусковыми механизмами ВСС являются жизнеопасные желудочковые аритмии (ЖЖА): желудочковая тахикардия (ЖТ) и фибрилляция желудочков (ФЖ), в основе патофизиологии которых лежит электрическая нестабильность миокарда, которая на клеточном уровне проявляется в форме нарушения проводимости кальциевых каналов мембран кардиомиоцитов [2,3]. В течение последнего десятилетия в клинических исследованиях и в эксперименте активно изучаются процессы изменений реполяризации (как на клеточном уровне, так и в миокарде в целом), потенциально являющиеся причиной ЖЖА: пространственная дисперсия реполяризации, альтернация клеточного потенциала действия, изменения в ионных токах. С помощью математического моделирования было продемонстрировано, что в определенных ситуациях кардиомиоциты могут находиться в устойчивом или мультистабильном равновесии, а при некоторых условиях - в неустойчивом состоянии $[4,5]$. При неадекватном изменении концентрации ионов $\mathrm{Ca}^{2+}$ происходит изменение длительности потенциала действия (ДПД) и периода рефрактерности. Если в соседних отделах миокарда изменения ДПД происходят в противофазе, возникает пространственная гетерогенность рефрактерного периода и формируются благоприятные условия для запуска механизма reentry. Явление гетерогенности ДПД отражается на ЭКГ в форме временной альтернации морфологии Т-зубца и пространственной дисперсии интервала QT.

Таким образом, электрическая нестабильность миокарда представляет собой пространственно-временное явление, сопряженное с феноменом электрической неустойчивости. Неустойчивые возбудимые среды в работах Л.Ландау и В.Гинзбурга трактуются с позиций нелинейной динамики. Этим объясняется, что в случае физиологической нормы пространственная волна возбуждения распространяется по миокарду без препятствий. Если же в миокарде имеются участки гетерогенности, могут возникать спиральные высокочастотные волны, аннигилирующие основную волну возбуждения, имеющую более низкую частоту. При этом создаются динамические волновые условия для пароксизмов желудочковой тахикардии и фибрилляции желудочков [2,3]. Кроме того, в нестабильных средах образуются точки бифуркации, в которых происходят резкие 
переходы, например, коэффициент передачи электрического импульса из режима 1:1 может скачкообразно перейти в режимы 1:2, 2:1, 4:3 и т.д. В совокупности все эти исследования создали основу для выявления маркеров электрической нестабильности миокарда - альтернации зубца Т и дисперсии интервала QT.

Самым распространенным неинвазивным методом для скринингового определения аритмического риска является холтеровское мониторирование (ХМ) электрокардиограммы (ЭКГ). Изучение изменений на ЭКГ, сопряженных с желудочковой эктопией, анализ их взаимосвязи с вегетативной регуляцией создает предпосылки для разработки новых способов диагностики риск-стратификационных маркеров. Так, в настоящее время одним из способов прогнозирования ВСС стала оценка показателей турбулентности сердечного ритма. Признаком нестабильности системы регуляции гемодинамики является патологическая турбулентность сердечного ритма (HRT - heart rate turbulence) [6]. HRT - это двухфазная физиологическая реакция синусового узла на желудочковую экстрасистолу (ЖЭС). Она состоит из короткого начального ускорения с последующим замедлением сердечного ритма. Патологическая HRT свидетельствует о дисфункции барорецепторного контроля и обладает выраженной прогностической значимостью. Так, по данным Е.В. Шляхто с соавт. 2-х летняя смертность у больных ИМ с патологической HRT в 3,5 раз выше, чем у таких же больных с нормальной HRT [7].

К независимым предикторам ВСС относят также синдром удлиненного QT и высокую дисперсию интервала QT (dispQT), которые считаются электрофизиологическим субстратом желудочковой тахикардии по типу “пируэт" $[8,9]$.

Таким образом, анализ параметров ЭКГ позволяет выявить ряд риск-стратификационных предикторов ВСС, объединенных в общее понятие "электрическая нестабильность", которая отражает временную и пространственную гетерогенность процессов реполяризации миокарда. Электрические процессы, связанные с гетерогенностью, проявляются на ЭКГ в виде альтернации Т-зубцов и дисперсии интервала QT, а барорецепторная дисфункция оценивается параметрами турбулентности HRT. Использование вышеперечисленных прогностических маркеров сердечной смерти имеет важное практическое значение, так как позволяет своевременно стратифицировать пациентов и выбирать оптимальную лечебную стратегию. Однако, внедрение этих методов оценки ЭКГ в нашей республике сдерживается от- сутствием специализированного программного обеспечения.

Целью работы является создание и клиническая апробация компьютерной программы для идентификации комплекса прогностических маркеров ВСС: альтернации Т-зубца, длительности и дисперсии интервала QT, турбулентности сердечного ритма (HRT) и изучение возможностей комбинированной оценки состояния автономной нервной системы для выявления электрической нестабильности миокарда у пациентов с кардиомиопатией (дилатационной, ишемической и гипертрофической) и риском развития желудочковых тахиаритмий.

\section{МЕТОДЫ}

Маркеры электрической нестабильности (HRT и TWA) определялись по данным анализа цифровой ЭКГ в 12 отведениях с длительностью записи 5 минут с помощью цифрового электрокардиографического комплекса «Интекард-7» (Минск). Предварительно проводилась адаптивная цифровая фильтрация сетевых, высокочастотных помех и дрейфа изолинии. Далее запись ЭКГ делилась на отрезки по 15 сек., в каждом из которых выполнялась оценка альтернации зубца T (TWA). Нами реализован алгоритм оценки TWA по методу бегущей средней с модификацией зоны поиска вероятной альтернации [10]. В зону поиска включали временной отрезок от точки J до точки, расположенной через 30 мс после максимума Т. В каждом кардиобите выделялся Т-зубец. Входной поток Т-зубцов разделили на четный и нечетный в каждом из которых выполняли процедуру выравнивания Т зубцов по максимумам. После чего подобные по форме Т-зубцы с коэффициентом корреляции >0,8 включали в анализ, остальные отбраковывали. По первым кардиобитам строили начальные усредненные четный и нечетный кардиобиты. Далее усредненные комплексы корректировались по алгоритму минимизации разности между ними путем прибавления или вычитания инкремента к текущему усредненному кардиобиту. Модифицированная бегущая средняя постоянно пересчитывалась по всему массиву. В итоге получали четный и нечетный усредненные кардиобиты. За локальную TWA принимали максимальную разность между ними. В качестве итоговой оценки TWA принимался абсолютный максимум из всех оценок, полученных в каждом из локальных 15-ти секундных с отрезков. Дополнительно рассчитывали Cv - коэффициенты вариации амплитуды и длительности TWA, вы- 


\section{Таблица 1 - Клиническая характеристика пациентов основной группы с жизнеопасными желудочковыми аритмиями}

\begin{tabular}{|c|c|c|c|c|}
\hline $\begin{array}{c}\text { Сердечно-сосудистое } \\
\text { заболевание }\end{array}$ & $\begin{array}{c}\text { Количество } \\
\text { пациентов } \\
(n=79)\end{array}$ & $\begin{array}{c}\text { Средний } \\
\text { возраст } \\
\text { (лет) }\end{array}$ & $\begin{array}{c}\text { XCH no NYHA } \\
\text { (ФK) }\end{array}$ & $\begin{array}{c}\text { Количество } \\
\text { пароксизмов ЖТ } \\
\text { по данным ХМ }\end{array}$ \\
\hline Ишемическая кардиомиопатия & 19 & $58,9 \pm 8,7$ & $2,9 \pm 0,9$ & $1,20 \pm 0,92$ \\
\hline Дилатационная кардиомиопатия & 33 & $48,8 \pm 9,3$ & $3,0 \pm 0,5$ & $2,30 \pm 1,67$ \\
\hline $\begin{array}{l}\text { Гипертрофическая } \\
\text { кардиомиопатия }\end{array}$ & 27 & $45,9 \pm 8,9$ & $1,9 \pm 0,8$ & $2,40 \pm 1,96$ \\
\hline
\end{tabular}

раженные в процентах. Низкой альтернации соответствовал Cv <15\%, умеренной - $15<\mathrm{Cv}<30 \%$, высокая (патологическая) альтернация устанавливалась при Cv>30\%.

Дисперсию QT в мс измеряли как разность между самым длинным и самым коротким интервалами QT в 10-ти последовательных комплексах ЭКГ-12 с усреднением показателя. Синдром высокой (патологической) дисперсии QT фиксировался при dispQT>70 мс.

Турбулентность сердечного ритма HRT в соответствии с международным стандартом оценивалась двумя параметрами: HRTO - начало турбулентности в \% и HRTS - наклон турбулентности в Mc/RR [6]. Физиологические значения нормы этих параметров соответствуют HRTO<0\% и HRTS $>2,5$ Mc/RR. Выделяли ситуации с одним или двумя патологическими параметрами HRT. Оценка HRT выполнялась, если в записи ЭКГ содержалась хотя бы одна желудочковая экстрасистола (ЖЭС) и 20 последующих синусовых сокращений сердца. При нарушении этих условий запись ЭКГ повторялась.

Последовательность работы компьютерной программы включала ввод идентификатора пациента, содержащего его семейный анамнез (синкопе, ИМ, АГ, сердечная недостаточность и\

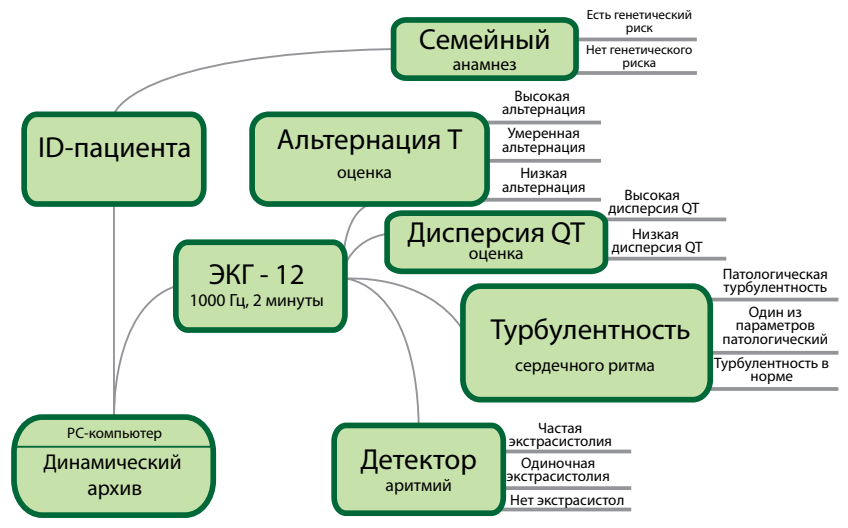

Рисунок 1 - Алгоритм программной оченки комплекса кардиологических маркеров электрической нестабильности миокарда или ВСС у родителей или ближайших родственников) и запись ЭКГ-сигнала, оцифрованного с частотой 1000 Гц, длительностью 5-7 минут. Далее по разработанным алгоритмам оценивался весь комплекс параметров электрической нестабильности миокарда, на базе которых основана риск-классификация ВСС (Рис.1). Все результаты заносились в динамический архив. Разработанная программа ориентирована на использование РС-компьютера и цифрового электрокардиографа "Интекард-7". Комплексное обследование пациентов также включало: эхокардиографию по общепринятой методике на ультразвуковом сканере Vivid 7 (GE,США), ХМ-ЭКГ на аппаратах «OXFORD Medilog Optima» (Великобритания) и «Phillips ZIMED» (Германия) на фоне базовой терапии.

\section{МАТЕРИАЛ}

Разработанная информационная технология апробирована в двух клинических группах исследуемых. Основная группа включала 79 па-

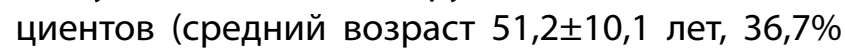
женщин) с выявленными при 24-часовом ХМ-ЭКГ жизнеопасными желудочковыми аритмиями (ЖЖА): пароксизмами устойчивой и неустой-

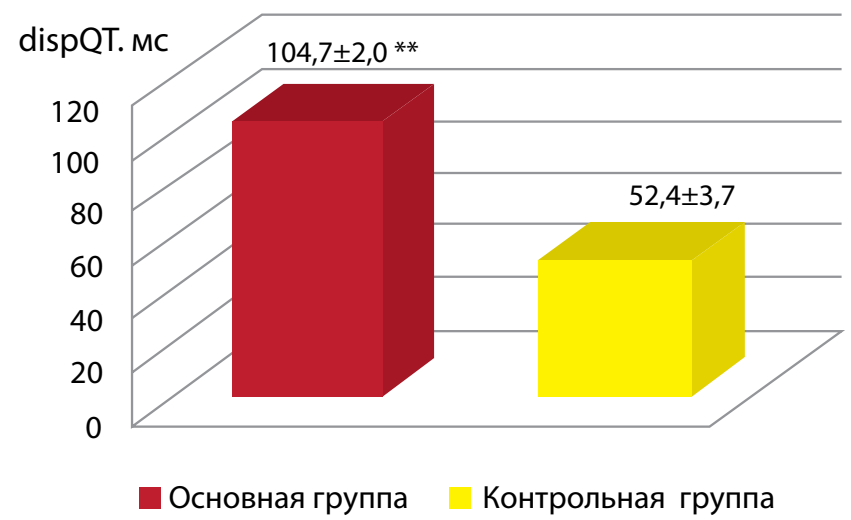

Рисунок 2 - Результаты оценки дисперсии интервала QTC в мс у больных с жезнеопасными желудочковыми аритмиями (основная групnа, $n=39$ ) и у больных без нарушений сердечного ритма (контрольная груп$n a, n=30),{ }^{*}$ - достоверность различий $p<0,001$ 


\section{Таблица 2 - Клиническая характеристика пациентов контрольной группы без желудочковых аритмий}

\begin{tabular}{|l|c|c|c|c|}
\hline Сердечно-сосудистое заболевание & $\begin{array}{c}\text { Количество } \\
\text { пациентов } \\
\text { (n=30) }\end{array}$ & $\begin{array}{c}\text { Средний } \\
\text { возраст } \\
\text { (лет) }\end{array}$ & $\begin{array}{c}\text { ХСН по } \\
\text { NYHА (ФК) }\end{array}$ & $\begin{array}{c}\text { Количество } \\
\text { пароксизмов ЖТ } \\
\text { по данным ХM }\end{array}$ \\
\hline Дилатационная кардиомиопатия & 15 & $51,2 \pm 10,1$ & $2,9 \pm 0,5$ & 0 \\
\hline Гипертрофическая кардиомиопатия & 15 & $49,2 \pm 11,3$ & $1,5 \pm 0,9$ & 0 \\
\hline
\end{tabular}

чивой желудочковой тахикардии (ЖТ). В контрольную группу включили 30 пациентов с КМП (средний возраст 50,7 79,8 лет, 33,3\% женщин), не имеющих желудочковой аритмии в анамнезе и при ХМ-ЭКГ. Клинические характеристики больных основной и контрольной группы представлены в таблицах 1 и 2.

Оценку желудочковой эктопической активности, анализ вариабельности и турбулентности сердечного ритма (HRT), альтернации Т-зубца и дисперсии интервала QT параллельно проводили по данным ХМ-ЭКГ и 5-минутной ЭКГ-12.

\section{РЕЗУЛЬТАТЫ}

В результате исследования выявили достоверные различия показателей турбулентности сердечного ритма, дисперсии QT и альтернации зубца Т (при отсутствии значимых отличий показателей ВСР) у больных с ЖЖА по сравнению с группой пациентов без клинически значимых аритмий. В основной группе среднее значение dispQT составило $104,7 \pm 2,0$ мс, а в контрольной группе пациентов без значимых нарушений рит-

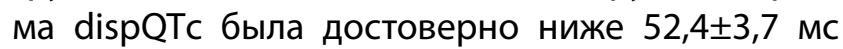
$(p<0,001)$. На рис. 2 представлены результаты оценки дисперсии интервала QTс в основной и контрольной группе. По оси ординат значения

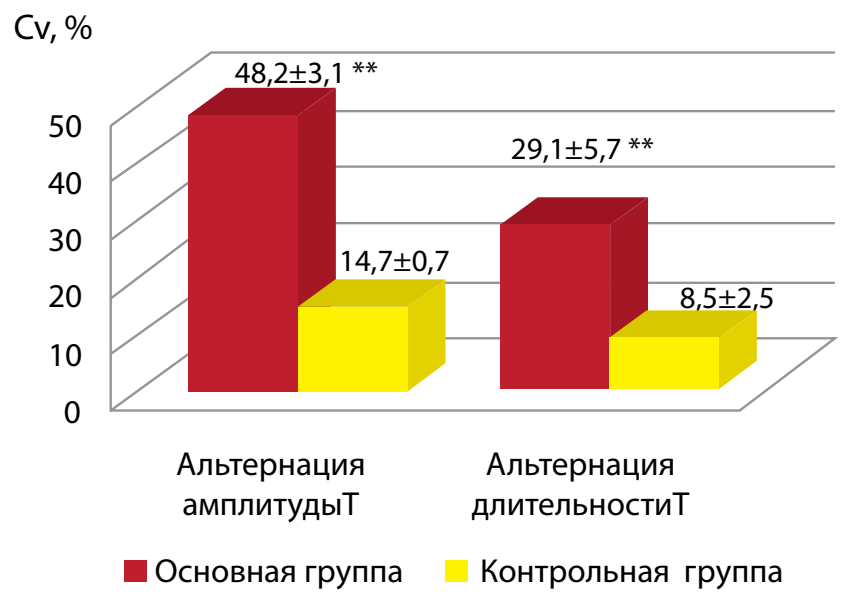

Рисунок 3 - Коэффициенты вариации альтернации Т-зубиа по амплитуде и длительности в \% у больных в основной $(n=39)$ и контрольной группе $(n=30)$, ** - достоверность различий $p<0,001$ дисперсии в мс. В основной группе dispQTс значительно превышает порог нормы (>70 мс), а в контрольной группе больных без зарегистрированных нарушений ритма dispQTс была достоверно ниже порога. Это свидетельствует о том, что у больных с ЖЖА ( $n=39)$ пространственная неоднородность процессов реполяризации миокарда достоверно выше нормы и отличается от контрольной группы.

У лиц без нарушений ритма альтернация амплитуды зубца Т по оценке коэффициента вариации составила $14,7 \%$, а длительности- 8,5\%, что указывает на качественную однородность совокупности признака. Напротив, у больных с ЖЖА альтернация амплитуды Т составила 48,2\% (высокий коэффициент альтернации), а длительности Т - 29,1\%, что отражает выраженную гетерогенность процессов реполяризации и электрическую нестабильность миокарда. Достоверность отличий с контрольной группой $p<0,001$. На рис. 3 представлены результаты оценки альтернации Т в основной и контрольной группах. По оси ординат отложены значения коэффициента вариации альтернации амплитуды и длительности зубца Т в \%. Полученные нами результаты оценки TWA согласуются с данными авторов исследования [3].

В основной группе пациентов с КМП и желудочковыми тахиаритмиями выявлены патологические значения ТCP (HRTO $=0,98 \pm 0,51 \%$ и $\mathrm{HRTS}=1,30 \pm 0,17 \mathrm{Mc} / \mathrm{RR})$, достоверно отличающиеся от показателей ТСР в контрольной группе пациентов без клинически значимых желудочковых аритмий (HRTO $=-3,2 \pm 0,27 \%$ и HRTS $=2,9 \pm 0,98$ $\mathrm{Mc} / \mathrm{RR}, \mathrm{p}<0,001)$. Патологический показатель «начало турбулентности»- HRTO- на 46,5\% чаще выявлялся в группе пациентов с ГКМП по сравнению с группой больных с ДКМП, где больше на $38,4 \%$ идентифицировался «наклон турбулентHости»- HRTS.

В таблице 3 систематизированы результаты сопоставления выявленных маркеров нестабильности TWA, dispQTс и HRT в основной и контрольной группах обследуемых. 


\section{Таблица 3 - Патологические изменения ЭКГ при ХМ-ЭКГ (Oxford) и 5-минутной ЭКГ (Интекард-7) в основной и контрольной группе пациентов}

\begin{tabular}{|l|c|c|c|c|c|}
\hline $\begin{array}{c}\text { Сравниваемые } \\
\text { группы }\end{array}$ & $\begin{array}{c}\text { Количество } \\
\text { пациентов }\end{array}$ & $\begin{array}{c}\text { Дисперсия } \\
\text { QТинтервала } \\
\text { (Mc) }\end{array}$ & $\begin{array}{c}\text { Альтернация } \\
\text { Т-зубца -TWA } \\
\text { (Cv\%) }\end{array}$ & $\begin{array}{c}\text { Турбулентность сердечного } \\
\text { ритма (HRT) (\%) }\end{array}$ \\
\hline Основная & 79 & $104,7 \pm 2,03$ & $48,2 \pm 3,12$ & $0,98 \pm 0,51$ & $1,3 \pm 0,17$ \\
\hline Контрольная & 30 & $52,4 \pm 3,71$ & $14,7 \pm 0,71$ & $-0,32 \pm 0,27$ & $2,9 \pm 0,98$ \\
\hline Достоверность, p & & $<0,001$ & $<0,001$ & $<0,001$ & $<0,001$ \\
\hline
\end{tabular}

\section{ОБСУЖДЕНИЕ}

TWA - показатель изменений формы, амплитуды, длительности или полярности зубца Т, электрокардиографический маркер уязвимости к ЖЖА, тесно связан с аритмогенезом и отражает изменения в электрофизиологических процессах сердца (так называемое электрическое ремоделирование). Полученные нами оценки TWA согласуются с Рекомендациями математической статистики [11]. Так у лиц без нарушений ритма альтернация амплитуды зубца Т составила 14,7\%, а длительности - 8,5\%, что указывает на качественную однородность совокупности признака. Можно считать, что процессы реполяризации в данном случае носят гомогенный, стабильный характер. Напротив, у больных с ЖЖА альтернация амплитуды Т составила 48,2\%, а длительности T - 29,1\%. Если коэффициент вариации Cv превышает $30 \%$, говорят о неоднородности и сильной вариации признака. Следовательно, в изученной нами группе больных с КМП и нарушениями ритма процессы реполяризации миокарда имеют выраженный гетерогенный характер, поэтому риск ВСС у таких больных существенно выше. Полученные нами данные на когорте пациентов $(\mathrm{n}=15)$ с ИКД, нуждаются в дальнейшем изучении и подтверждении возможности применения и оптимизации теста TWA с выявленияем специфического порога (количественного и качественного) для отбора пациентов, нуждающихся в профилактической ИКД-терапии. Применение этой методики особенно необходимо для пациентов КМП не только с высоким риском ВСС, но и без выявления предшествующих эпизодов ЖЖА. Установлен интересный для практического применения факт: 4-ем пациентам с ГКМП и 11-и больным из основной группы по клиническим показаниям (класс показаний I) были имплантированы кардиовертеры-дефибрилляторы (ICD) или ресинхро-низирующие устройства с функцией дефибриллятора (CRT-D). При ретроспективной оценке результатов TWA в этой группе пациентов выявлена высокая патологическая

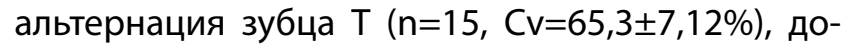
стоверно $(p<0,001)$ отличающаяся от таковой в остальной группе (ДКМП+ГКМП) с ЖЖА (n=60, $C v=33,5 \pm 8,52 \%)$, имеющих также патологические значения этого показателя.

В отношении дисперсии QT ситуация такова. У больных с нарушениями ритма среднее значение dispQT превысила пороговое значение "норма-патология" на 49,6 \%, а у больных без нарушений сердечного ритма среднее dispQT оказалось ниже порога на 25,1\%. Полученные данные подтверждают возможность использования и ценность показателя дисперсии интервала QTс в качестве предиктора ЖЖА.

TPC отражает эффективность барорефлекторных механизмов автономной регуляции ритма к быстрой компенсации внутрисердечных гемодинамических дисфункций, вызванных желудочковыми аритмиями. При нарушенном автономном контроле ритма сердца эта реакция ослаблена или полностью отсутствует, что подтверждается нашими исследованиями. Взаимосвязи показателей HRTO и HRTS не отмечалось, но чаще патологический показатель «начало турбулентности» ТСР встречался у пациентов с ГКМП, а «наклон турбулентности» ТСР - у больных с ДКМП.

\section{Выводы}

Выполненное исследование свидетельствует о том, что в клинической практике для скрининговой процедуры выявления предикторов жизнеопасных желудочковых аритмий (альтернация зубца Т, длительность и дисперсия интервалов QTc, турбулентность сердечного ритма) может быть использован программно-алгоритмический комплекс "Интекард-7". Полученные результаты исследования продемонстрировали достоверные различия показателей турбулентности сердечного ритма, дисперсии QTc, TWA-альтернации зубца Т у больных с КМП и жизнеопасными желудочковыми нарушениями ритма по сравнению 
с группой пациентов без клинически значимых аритмий. Нами установлено, что у пациентов с ЖЖА альтернация амплитуды Т зубца в 3,3 раза превышает этот показатель в группе без аритмий, турбулентность сердечного ритма (HRTO) в 3 раза, а дисперсия QTс в 2 раза выше, чем аналогичные показатели в контрольной группе пациентов без клинически значимых нарушений сердечного ритма.

Таким образом, полученные данные подтверждают возможность использования электрокардиографических критериев: ТСР, альтернации Т зубца и дисперсии QTс в качестве предикторов ВСС с целью своевременной стратификации риска желудочковых тахиаритмий и выбора оптимальной лечебной тактики.

\section{СПИСОК ЛИТЕРАТУРЫ}

1. Radoi N., Pescariu S., Ivanica G. et al. Microvolt T-wave alternans: a new method to evaluate the risk of sudden cardiac death. Timisoara medical J. 2004; 2: 179-183.

2. Karma A., Gilmour R. Nonlinear dynamics of heart rate disorders. Physics Today 2007; 3: 51-57.

3. Zheng I., Colleen E. L-type Ca2+ channel mutation and T-wave alternans: a model study. American J. of Physiology 2007; 292: 66-75.

4. Суровяткина Е.Д. Методы нелинейного анализа в динамике электрической активности сердечной клетки человека. В: Назиров Р.Р. (ред.). Методы нелинейного анализа в кардиологии и онкологии: физические подходы и клиническая практика. Вып.2. М: КДУ, 2010: 206 с.

5. Janse M. Is there chaos in cardiology? British Heart J. 1992; 67:3-4.

6. Bauer A., Malik M., Schmidt G. et al. Heart rate turbulence: standards of measurement, physiological interpretation and clinical use. Amer. Coll. Cardiology 2008; 52: 1353-56.

7. Шляхто Е.В., Бернгардт Э.Р., Пармон Е.В. и др. Турбулентность сердечного ритма в оченке риска внезапной сердечной смертности. Вестник аритмологии 2005; 38: 49-55.

8. Мрочек А.Г., Гончарик Д.Б., Часнойть А.Р. и др. Синдром удлиненного QT . Кардиология в Беларуси 2010; 3: 28-23.

9. Kautzer J., Malic M. QT interval dispersion and its clinical utility. Pac. Clin. Electrophysiol. 1997; 20(10): 2625-2640.

10. Khaustov A., Nemati S., Clifford G. An opensource standard T-wave alternans detector for benchmarking. Computers in Cardiology 2008; 52: 509- 512.

11. Лыпчук В.В., Лысюк О.В. Методические рекомендации из статистики. - Львов: ЛДАУ, 2009. $-93 c$. 